\title{
Mutant p53 Gain-of-Function in Cancer
}

\author{
Moshe Oren and Varda Rotter \\ Department of Molecular Cell Biology, The Weizmann Institute, Rehovot 76100, Israel \\ Correspondence: moshe.oren@weizmann.ac.il
}

In its wild-type form, p53 is a major tumor suppressor whose function is critical for protection against cancer. Many human tumors carry missense mutations in the TP53 gene, encoding p53. Typically, the affected tumor cells accumulate excessive amounts of the mutant p53 protein. Various lines of evidence indicate that, in addition to abrogating the tumor suppressor functions of wild-type p53, the common types of cancer-associated p53 mutations also endow the mutant protein with new activities that can contribute actively to various stages of tumor progression and to increased resistance to anticancer treatments. Collectively, these activities are referred to as mutant p53 gain-of-function. This article addresses the biological manifestations of mutant p53 gain-of-function, the underlying molecular mechanisms, and their possible clinical implications.

$M$ utations in the TP53 gene, encoding the p53 tumor suppressor, are arguably the most frequent type of gene-specific alterations in human cancer. This attests to the centrality of p53 as a major mainstay in the body's built-in anticancer defense mechanisms. Not surprisingly, this pivotal role of the wild-type p53 (wtp53) protein in tumor suppression has attracted many researchers to study it in detail, resulting in an avalanche of information and publications. One might expect that, similar to other tumor suppressor genes, the sole outcome of mutations in the TP53 gene will be loss of wtp53 function, characteristically manifested as total lack of p53 expression or production of unstable or truncated mutant proteins. Yet, quite strikingly, the vast majority of cancerassociated p53 mutations actually lead to production of full length protein, typically with only a single amino acid substitution, which tends to accumulate in the tumor cells and reach steady-state levels that greatly exceed those of wtp53 in noncancerous cells (Rotter 1983). This remarkable feature has suggested early on in p53 research that cancer-associated mutant p53 (mutp53) isoforms may be more than just relics of wtp53 inactivation, and may instead play distinctive roles in the tumor cells.

In principle, emergence of a p53 mutation within a cell might have three, not mutually exclusive, types of outcome (Michalovitz et al. 1991; Sigal and Rotter 2000; Weisz et al. 2007b). First, such mutation is expected to abrogate the tumor suppressor function of the affected TP53 allele, reducing the overall capacity of the cell to mount a proper p53 response; if both alleles eventually become mutated, or if the remaining allele is lost, such cells will be

Editors: Arnold J. Levine and David P. Lane

Additional Perspectives on The p53 Family available at www.cshperspectives.org

Copyright (C) 2010 Cold Spring Harbor Laboratory Press; all rights reserved; doi: 10.1101/cshperspect.a001107

Cite this article as Cold Spring Harb Perspect Biol 2010;2:a001107 
totally deprived of anticancer protection by p53. Second, many common mutp53 isoforms can exert dominant-negative effects over coexpressed wtp53, largely by forming mixed tetramers that are incapable of DNA binding and transactivation. Hence, even if one wt allele is retained, the cell may be rendered practically devoid of wtp53 function through such mechanism, particularly if the mutant protein is expressed in excess over its wt counterpart. Third, and most relevant for this article, the emergent mutp53 protein might possess activities of its own, often not present in the original wtp53 protein, which can actively contribute to various aspects of tumor progression. Such activities, commonly described as mutp53 gain-of-function (GOF), are the subject of this article. Several recent reviews address in detail the various aspects of mutp53 GOF (Brosh and Rotter 2009; Donzelli et al. 2008; Lozano 2007; Olivier et al. 2009; Peart and Prives 2006; Petitjean et al. 2007; Song and Xu 2007; Strano et al. 2007; Weisz et al. 2007b). Therefore, we focus here mainly on general principles as well as on some of the more recent findings.

\section{DEFINITION OF mutp53 GOF}

Even though the idea of mutp53 GOF is fairly simple, the exact definition of what constitutes a true GOF of mutp53 is not always straightforward. Technically, GOF is often monitored by overexpressing a particular mutp53 isoform and measuring its impact on the properties of the overexpressing cells, be it in culture or in mouse tumor models. As discussed previously, many p53 mutants have dominant-negative effects over wtp53. Hence, effects obtained by overexpression of mutp53 in cells that harbor endogenous wtp53 do not necessarily show the existence of a GOF. To rigorously prove GOF, one thus has to perform such experiments in cells that are otherwise p53-null, an approach that is now common practice in the field. A complementary approach to prove mutp53 GOF, which has become feasible by the advent of siRNA technology, relies on knocking down endogenous mutp53 in tumorderived cells that harbor naturally occurring p53 mutations and monitoring changes in cell phenotype.

In that regard, one also has to consider the demonstrated ability of many mutp53 isoforms to bind and inactivate the p53-related proteins p63 and p73. As discussed later, this is a pivotal mechanism for mutp $53 \mathrm{GOF}$. If one wishes to be extrarigorous, it could be argued that this is not a true GOF, because the activities of p63 and p73 that are quenched by mutp53 are often quite akin to those of wtp53 itself, including the induction of common target genes. Yet, we do not adopt this extreme view, and adhere to the definition that GOF encompasses any activity of mutp53 exerted in the absence of coexpressed wtp53.

\section{HISTORY OF mutp53 GOF RESEARCH}

The concept of mutp53 GOF was formally introduced in 1993, when it was shown that mutp53 isoforms of both human and mouse origin, but not wtp53, can transform p53-null cells and endow them with an increased ability to form colonies in soft agar in vitro and tumors in mice (Dittmer et al. 1993). Yet, the truth of the matter is that mutp53 GOF was experimentally demonstrated already many years earlier, except that the results could not be properly interpreted at that time. This is yet another reflection of the unusual history of p53 research: The first p53 cDNA clones, isolated by several laboratories, all originated from tumor-derived or in-vitro-transformed cells (Levine and Oren 2009). As is now common knowledge, such cells often tend to harbor p53 mutations. This was also the case for the cells employed to obtain the first p53 cDNA clones. Thus, the p53 expression vectors employed in the early biological experiments encoded various mutp53 isoforms rather than wtp53. Consequently, although researchers thought they were monitoring the effects of wtp53, they were actually studying those of mutp53. Not surprisingly, these experiments led to the erroneous conclusion that p53 was an oncogene, an issue resolved only years later, on proper examination of the biological activities of bona fide wtp53. These subsequent studies also clarified that the oncogenic effects observed 
earlier, which led to the misclassification of $\mathrm{p} 53$ as an oncogene, were unique to mutp53 but not wtp53.

Many of those early studies were based on transfection of p53 expression plasmids into nontransformed cells that express endogenous wtp53; hence, the observed effects of mutp53 might have been caused by a combination of dominant-negative action and GOF. Yet, in some of those studies, the target cells were indeed p53-null, and thus the effects of mutp53 overexpression are ascribable to true mutp53 GOF. In retrospect, the first experimental manifestation of mutp53 GOF was therefore in 1984, in a study in which p53-deficient Abelson murine leukemia-transformed cells were rendered highly tumorigenic in vivo by overexpression of a plasmid that actually encoded mutp53 (Wolf et al. 1984). Nevertheless, the study of Dittmer and coworkers (Dittmer et al. 1993), as well as reports on the ability of mutp53 to exert unique transcriptional effects (Chin et al. 1992; Deb et al. 1992; Matas et al. 2001), ushered in officially the era of mutp53 GOF research.

\section{BIOLOGICAL MANIFESTATIONS OF mutp53 GOF IN CULTURED CELLS}

By and large, our current knowledge about mutp53 GOF draws heavily on experiments in which various mutp53 isoforms have been overexpressed in p53-null cells, although this is being increasingly bolstered in recent years by siRNA-mediated knockdown studies. Although forced overexpression represents an artificial situation and thus has to be viewed with great caution, it is probably more justified in the case of mutp53. After all, the hallmark of p53 alterations in cancer is the vast overproduction of mutp53 proteins, which is often exacerbated during tumor progression. In the following discussion, we discuss the main biological manifestations of mutp53 GOF.

\section{Mutp53 and Genomic Instability}

A major hallmark of cancer progression is a gradual increase in genome instability, manifested all the way from higher mutation rates to gross aberrations in chromosome number and structure. A link between mutp53 and increased genomic instability was clearly demonstrated by showing that human mutp53 can disrupt normal spindle checkpoint control, leading to accumulation of cells with polyploid genomes (Gualberto et al. 1998). Additional studies revealed further manifestations of the enhancement of genomic instability by mutp53, as reflected by higher mutation rates in the T-cell receptor of cells exposed to Xirradiation (Iwamoto et al. 1996), increased frequency of centrosome amplification and aberrant mitoses in mouse mammary epithelial cells (Murphy et al. 2000), as well as increased gene amplification in Saos2 cells (El-Hizawi et al. 2002). This link was extended to an in vivo context, revealing that expression of the mouse equivalents of human "hotspot" p53 mutants results in tumors that exhibit a high degree of genomic instability, manifested by aneuploidy associated with aberrant centrosome amplification as well as nonreciprocal chromosome translocations without evidence of telomere erosion (Caulin et al. 2007; Hingorani et al. 2005).

As discussed later, many GOF effects of mutp53 rely on its ability to bind and inactivate the p53-related proteins p63 and p73. In that regard, it is noteworthy that in p53-deficient cells, p73 can replace p53 in maintaining genome stability by suppressing aneuploidy and polyploidy (Talos et al. 2007). Abrogation of p73 function by excess mutp53 is thus likely to augment the accumulation of cells with polyploid genomes and aberrant chromosome numbers, thereby facilitating cancer progression. This might explain, at least partially, the early observations of Gualberto et al. (1998). In addition, mup53 can interfere with DNA repair by attenuating base excision repair (Offer et al. 1999).

The ability of mutp53 to disrupt mechanisms that maintain cellular genome integrity might provide an appealing explanation for its impact on tumor progression, particularly in advanced stages of the disease in which gross manifestations of genomic instability are very frequent. 


\section{Mutp53 and Antiapoptosis}

One distinctive feature of many p53 GOF mutants is an ability to confer on cells an elevated resistance to a variety of proapoptotic signals. This effect of mutp53 was revealed already in 1995, by showing that mutp53 can suppress cMyc-induced apoptosis in leukemic cells (Lotem and Sachs 1995). Because aberrantly elevated expression of cMyc is a frequent event in many types of cancer, this finding suggests that p53 mutations might enable tumor cells to benefit from the pro-proliferative effects of cMyc without paying the price tag of cMyc-induced apoptosis. Subsequent studies provided further insight into the antiapoptotic effects of mutp53. Thus, mutp53 could protect cells against apoptosis induced by growth factor deprivation (Peled et al. 1996), suggesting a mechanism whereby p53 mutations confer a selective advantage on aspiring tumor cells within a competitive microenvironment.

Of particular interest, overexpression of various tumor-associated mutp53 isoforms can render cells markedly more resistant to killing by a variety of anticancer agents, whereas knockdown of endogenous mutp53 sensitizes cancer cells to killing by such agents. Thus, mouse cells expressing a murine mutp53 isoform showed increased resistance to $\gamma$ irradiation, doxorubicin, and cisplatin (Li et al. 1998). A similar effect on resistance to etoposide and cisplatin was observed in human H1299 lung cancer cells overexpressing different types of tumor-associated human p53 mutants, notably p53R175H and p53R273H (Blandino et al. 1999). An antiapoptotic role of experimentally overexpressed mutp53 was also demonstrated by many additional studies (Matas et al. 2001; Murphy et al. 2000; Yap et al. 2004). The biological relevance of these findings was later confirmed by showing that siRNA-mediated knockdown of endogenous mutp53 in cancer-derived cell lines rendered them more vulnerable to apoptotic cell death induced by anticancer agents and other proapoptotic stimuli (Vikhanskaya et al. 2007; Weisz et al. 2007a; Weisz et al. 2004; Wong et al. 2007), a finding extended also to an in vivo setting (Bossi et al. 2006). The antiapoptotic activities of mutp53 may thus not only accelerate tumor progression but also hinder the response of cancer patients to anticancer therapy. This does not necessarily imply that cancers with p53 mutations will be more refractory to therapy (see Olivier et al. 2010). Conceivably, in tumors lacking p53 mutations, alternative mechanisms may exist that are equally potent in rendering the cells chemoresistant. Yet, in those tumors that do harbor p53 mutations, ablation of mutp53 GOF activity might facilitate their killing by chemotherapy.

\section{Mutp53 and Cell Migration and Invasion}

The impact of p53 mutations on tumorigenesis probably depends on many factors, including the stage in the process when p53 mutations and mutp53 accumulation occur. At least in some types of cancer, p53 mutations are rather late events, correlating with progression to aggressive, advanced disease (Vogelstein and Kinzler 1993). Remarkably, as discussed later, increased tumor aggressiveness and higher metastatic potential are also hallmarks of mutp53 GOF in mouse models. These advanced stages in tumor progression are characterized by acquisition of an ability of the cancer cells to invade adjacent tissue, migrate toward distant sites, and seed metastases. In cultured cells, these features can be addressed by monitoring cell migration (e.g., by "wound healing" assays in which some of the cells are scratched off the dish or in transwell assays in which cells are asked to migrate across a membrane) and cell invasion (e.g., through a layer of extracellular matrix). Indeed, recent work indicates that mutp53 can augment cell migration and invasion in such in vitro assays (Adorno et al. 2009; Wang et al. 2009). Although published information on this aspect of mutp53 GOF is still scanty, it is likely to rapidly become a focal area of mutp53 research, given its obvious relevance to the knowledge gained from clinical data and in vivo mouse tumor model analysis.

Induction of cell migration by mutp53 is highly cell-context-dependent, and additional signals such as oncogenic Ras in combination with TGF- $\beta$ might be required to unleash this 
activity (Adorno et al. 2009). Indeed, gene expression profiling studies reveal that cancerassociated mutp53 isoforms cooperate synergistically with oncogenic Ras to induce a procancerous gene cluster consisting of chemokines, interleukins, and ECM-related molecules, which are major contributors to tumor progression and invasion (Buganim et al., unpubl. data). This requirement for cooperating oncogenic signals may explain why other studies did not find a positive role for mutp53 GOF in promoting migration (Dong et al. 2007), or even found a negative role (Kalo et al. 2007). Furthermore, in cells that retain a wtp53 allele, the promigratory effect of mutp53 might be exerted through a combination of GOF and dominant-negative activities (Dong et al. 2007).

\section{Mutp53 and the TGF- $\beta$ Pathway}

Transforming growth factor $\beta$ (TGF- $\beta$ ) has a dual impact on cancer progression. In early stages of the process, it serves as a tumor suppressor, strongly inhibiting the proliferation of epithelial cells; however, at advanced stages of the disease, it can turn into a potent driver of tumor spread and metastasis, severely worsening patient prognosis (Derynck et al. 2001; Massague 2008). Previous work has shown that the antiproliferative effect of TGF- $\beta$ is facilitated by the presence of wtp53, testifying to the cooperation of these two pivotal signaling pathways in suppression of early stages of tumor progression (Cordenonsi et al. 2003). More recent investigation of the cross talk between mutp53 and TGF- $\beta$ has revealed an interesting and intriguing duality also here. On the one hand, mutp53 can repress the expression of TGF- $\beta$ receptor type II, thereby attenuating TGF- $\beta$-mediated signaling (Kalo et al. 2007). However, in a number of experimental models, mutp53 was actually found to augment the promigratory, proinvasive, and prometastatic properties of TGF- $\beta$ both in vitro and in vivo (Adorno et al. 2009). These seemingly inconsistent findings are well in line with the very different facets of TGF- $\beta$ in tumor progression. It thus is conceivable that if p53 mutations occur in epithelial cells relatively early, they will indeed accelerate cell proliferation and contribute to tumor progression by overriding the inhibitory actions of TGF- $\beta$ as a tumor suppressor. In contrast, if p53 mutations occur late in the process, when cells are no more susceptible to the antiproliferative effects of TGF- $\beta$, augmentation of its proinvasive effects is likely to render the tumors more aggressive and worsen patient outcome. One still needs to explain, however, why mutp53 modulates TGF- $\beta$ activity differently at different stages of tumor progression. A likely mechanistic explanation, although probably not the only one, is provided by the finding that the cooperation between mutp53 and TGF- $\beta$ in augmenting cell migration and metastasis is strongly enhanced by the presence of oncogenic mutant Ras (Adorno et al. 2009). Because Ras mutations are often associated with tumor progression and conversion of TGF- $\beta$ from a tumor suppressor into a prometastatic factor (reviewed in Derynck and Akhurst 2007), it is tempting to speculate that they also serve as the switch that alters the biological outcome of the interactions between mutp53 and TGF- $\beta$.

\section{Other Biological Effects of mutp53}

It is most certain that mutp53 GOF may manifest itself in a variety of additional ways, probably depending on cell context. Not surprisingly, overexpressed mutp53 can augment cell proliferation, which is apparent both in culture and in mice (Deb et al. 2002; Duan et al. 2008; Haupt et al. 2009; Scian et al. 2004b). As mutp53 research moves into new arenas, we might expect to find it involved in many additional activities that are typical of cancer cells such as energy metabolism, various biosynthetic pathways, inflammatory responses, and much more. Many of those activities are modulated by wtp53; it is reasonable to predict that, at least in many cases, mutp53 will exert an opposite effect.

\section{ANIMAL MODELS FOR mutp53 GOF}

Animal models are playing an increasingly central role in elucidating the biological manifestations 
of mutp53 GOF. As already mentioned, overexpression of mutp53 was shown long ago to contribute to tumorigenesis in mice (Wolf et al. 1984). In later studies, several hotspot p53 mutants were found to enhance tumor formation in nude mice when overexpressed in p53-null mouse fibroblasts, human p53-null osteosarcoma cells, or T cell leukemia-derived cells (Dittmer et al. 1993; Hsiao et al. 1994; Lanyi et al. 1998; Taylor et al. 1992), firmly establishing the generality of mutp53 GOF. Furthermore, transgenic mouse models driving tissue-specific mutp53 overexpression revealed a variety of manifestations of mutp53 GOF, such as cooperation with additional oncogenic events in accelerating tumor development and in rendering the tumors more invasive and aggressive (Duan et al. 2002; Duan et al. 2009; Heinlein et al. 2008). Of particular interest, overexpressed mutp53 was found to augment experimental metastasis in mice (Pohl et al. 1988), a feature recapitulated in a transgenic mouse model (Heinlein et al. 2008).

Although one could argue that the previous findings are caused by artificial overexpression of mutp53, their validity was shown by two additional, complementary approaches, namely knockdown of endogenous mutp53 in tumorderived cells and production of mutp53 knockin mice. Thus, using either stable or conditional shRNA-mediated knockdown of mutp53 in a number of human cancer cell lines implanted in nude mice, Bossi and coworkers showed that down-regulation of the endogenous mutp53 rendered those cells significantly less tumorigenic (Bossi et al. 2006; Bossi et al. 2008). In agreement with the impact of mutp53 on the response to genotoxic anticancer drugs in vitro, such knockdown sensitized the tumors to chemotherapy in vivo (Bossi et al. 2006). Remarkably, the tumors generated following mutp53 knockdown were less vascularized, suggesting a positive role of mutp53 in regulation of angiogenesis (Bossi et al. 2008). Furthermore, whereas knockdown of endogenous mutp53 in MDAMB-231 human breast cancer cells did not affect primary tumor growth, it strongly reduced metastasis to both lymph nodes and lung (Adorno et al. 2009).
The generation of mutp53 knockin mice, in which the endogenous wtp53 allele was replaced by mutant versions mimicking common human hotspot mutations, was perhaps the most significant advance in mutp53 research in recent years. These mice were modeled after the human Li-Fraumeni syndrome, in which germline p53 mutations confer a highly elevated susceptibility to succumb to early onset cancer (Malkin et al. 1990; Srivastava et al. 1990). The first two knockin studies, published in parallel in 2004, showed that when compared with p53 knockout animals, mice carrying mutp53 alleles tended to develop more aggressive, metastatic tumors, as well as a higher frequency of tumor types that are associated with p53 mutations in human cancer (Lang et al. 2004; Olive et al. 2004). When assayed in a skin carcinogenesis model driven by oncogenic mutant K-Ras, such mice exhibited increased tumor formation, accelerated tumor progression, and elevated rates of metastasis relative to their p53-null counterparts (Caulin et al. 2007). Very similar effects of combining endogenous mutp53 with endogenous oncogenic K-Ras were found in a mouse model of pancreatic cancer, where this combination led to a very invasive, widely metastatic disease (Hingorani et al. 2005). In both cases, the tumors displayed extensive genomic instability. By showing that animals expressing mutp53 proteins at physiological levels and under physiological regulation exhibit distinct cancer-associated features, these studies endowed mutp53 GOF research with the credibility that it had been somewhat lacking till then.

\section{BIOCHEMICAL BASIS OF mutp53 GOF}

The majority of tumor-associated p53 mutations, particularly those defined as mutational "hotspots," occur within the DNA binding domain (DBD) of p53 (see Olivier et al. 2010). Broadly speaking, these mutations can be roughly divided into two structural subgroups: DNA contact mutants (exemplified by the hotspot mutant p53R273H), affecting residues directly involved in sequence-specific DNA contacts without altering the overall conformation of 
the p53 molecule, and conformational mutants (exemplified by hotspot mutant p53R175H), in which the mutation leads to partial or complete abrogation of the wt conformation of the DBD, exposing residues and interfaces that are normally buried within the DBD. The current thinking is that wtp53 might also adopt such "mutant" conformation on occasion, perhaps in response to particular proliferative signals, but cancer-associated mutations "fix" the protein in the altered conformational state and prevent it from engaging in sequence-specific interactions with DNA elements recognized by wtp53 (Joerger and Fersht 2007; Joerger and Fersht 2008). Given the profound differences between the DNA contact mutants and conformational mutants, one might expect that they should operate through very distinct mechanisms and differ greatly in their impact. Indeed, quantitative differences between the effects of representative members of the two classes can easily be revealed in culture and in animal models (Dittmer et al. 1993; Halevy et al. 1991), and may also have prognostic significance (see Olivier et al. 2010). Yet, despite the quantitative differences, the two classes of mutants exhibit many qualitative similarities in their biochemical mode of action.

The term "gain-of-function" seems to imply that mutp53 acts through mechanisms that are totally uncharted by wtp53. However, this is not necessarily the case. Rather, at least some of the biochemical activities of mutp53 might stem from its having lost sequence-specific DNA binding while retaining the functionality of other domains. For instance, cancer-associated mutp53 proteins typically retain an intact transactivation domain (TAD), which may still operate exactly as it does within the wtp53 protein, but can now be targeted to different sites on the chromatin. Furthermore, given the high concentration of mutp53 protein in tumor cells, relatively weak molecular interactions, which are marginal within the wtp53 protein, may now be amplified by mass action and reach a threshold that allows them to exert a measurable impact on biochemical processes within the cell.

When expressed at sufficiently high levels, tumor-associated mutp53 isoforms can exert profound effects on gene expression patterns, thereby promoting specific biological outcomes while disfavoring others. Attempts to identify mutp53-regulated genes, initially by educated guesses and later by expression microarray analysis, yielded an ever growing list of candidates, some of which have been validated and shown to have functional relevance to mutp53 GOF. Many of those genes are associated in various ways with cell proliferation, tumor progression, and cancer biology in general, in line with the biological effects of mutp53. Among the first genes found to be up-regulated by mutp53 were the multi drug resistance 1 gene (MDR1) (Chin et al. 1992), PCNA (Deb et al. 1992), EGFR (Ludes-Meyers et al. 1996), IGF1R (Werner et al. 1996), c-myc (Frazier et al. 1998), and IGF2 (Lee et al. 2000), as well as genes encoding several ribosomal proteins (Loging and Reisman 1999). Subsequent studies revealed that mutp53 can up-regulate genes encoding many additional proproliferative or antiapoptotic proteins such as NFKB2, encoding the $\mathrm{p} 52$ subunit of NF-kB (Deb et al. 2002; Scian et al. 2005; Weisz et al. 2007a), Egr1 (Weisz et al. 2004), cyclin A, cyclin B1, cdk1, cdc25C (Di Agostino et al. 2006), calmodulin 2 (Knaup and Roemer 2004), hTERT (Scian et al. 2004a), stathmin (Singer et al. 2007), and Galectin-3 (Lavra et al. 2009) (see Brosh and Rotter 2009 for comprehensive review). Furthermore, just like wtp53, mutp53 can not only up-regulate specific genes but also repress the transcription of others, some examples being the proapoptotic CD95/Fas/Apo1 (Gurova et al. 2003; Zalcenstein et al. 2003), ATF3 (Buganim et al. 2006), TGFR2 (Kalo et al. 2007), caspase-3 (Wong et al. 2007), inhibitor of differentiation 2 (Id2) (Yan et al. 2008), and classical wtp53 target genes such p21, gadd45, PERP, and PTEN (Vikhanskaya et al. 2007). The palette of genes that can be modulated by mutp53 is probably much larger. In particular, given that mutp53 can interact with a variety of transcription factors (see later), often in a signaldependent manner (e.g., Adorno et al. 2009; Di Agostino et al. 2006; Weisz et al. 2007a), the subset of genes affected by mutp53 is likely to vary greatly among different cell types and cell contexts. 


\section{Oren and V. Rotter}

Mechanistically, the transcriptional effects of mutp53 are largely mediated by either of two types of molecular interactions (Fig. 1). On the one hand, mutp53 can physically interact with various isoforms of the p53-related proteins p63 and p73 and alter their transcriptional activity, typically resulting in negation of $\mathrm{p} 63 / \mathrm{p} 73$ function. On the other hand, mutp53 can engage in protein-protein interactions with a growing number of transcription factors, often being recruited to binding sites of those factors on chromatin, and modulate their transcriptional output both positively and negatively.

The p63 and p73 genes each encode numerous isoforms, derived by a combination of multiple transcription start sites and alternative splicing; some of those isoforms, particularly those that retain a functional amino-terminal transactivation domain (TA isoforms), can mimic wtp53 to some extent and activate a subset of target genes shared with wtp53, owing to the high similarity between the DBDs of all three members of the p53 family. However, both p63 and p73 also possess additional, distinct activities, which are not shared with wtp53 and impinge on many important biological processes, most notably development and differentiation (see Beckerman and Prives 2010). At least some cancer-associated mutp53 proteins can engage in direct protein - protein interactions with some, albeit not all, p73 and p63 isoforms, rendering them transcriptionally inactive (Gaiddon et al. 2001; Marin et al. 2000; Strano et al.2002). Consequently, genes that are normally controlled by $\mathrm{p} 63$ or p 73 in a given cell will become deregulated. Depending on whether $\mathrm{p} 63 / \mathrm{p} 73$ regulate a particular gene positively or negatively, mutp53 overexpression will result in repression or induction of that gene, respectively. Various

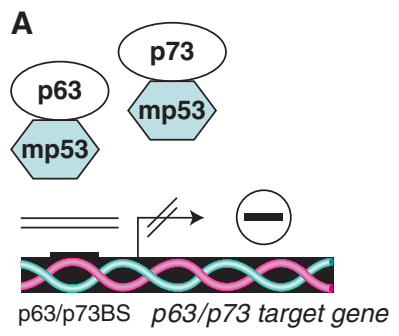

C

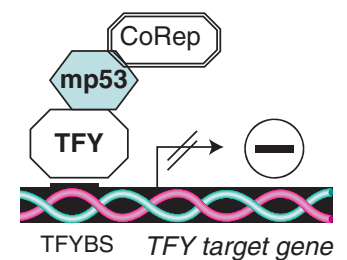

B

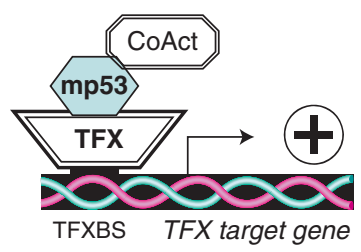

D

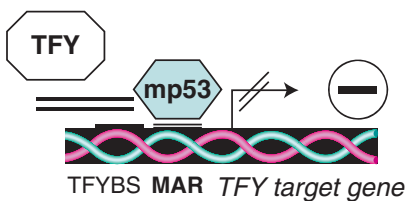

Figure 1. Mechanisms of transcriptional regulation by mutp53. Positive and negative effects on transcription are indicated by + and - , respectively. In $(A)$, mutp53 binds to $\mathrm{p} 63$ and/or $\mathrm{p} 73$ protein isoforms, inhibiting their interaction with cognate binding sites on the DNA ( $633 / \mathrm{p} 73 \mathrm{BS}$ ) and blocking the activation of p63/p73 target genes. In (B), mutp53 engages in protein-protein interactions with transcription factor X (TFX) and is tethered to the binding site of TFX on DNA; through its TAD, mutp53 recruits transcriptional coactivators (CoAct) such as p300 (Di Agostino et al. 2006), and augments transcription of TFX target genes. In $(C)$, mutp53 is proposed to be tethered to DNA through transcription factor Y (TFY), but instead of recruiting transcriptional coactivators it recruits corepressors, as has been shown for wt p53 (Murphy et al. 1999); what dictates whether mutp53 will recruit coactivators or corepressors is presently unknown, but in both $(B)$ and $(C)$, mutp53 will be found on chromatin at the appropriate TF binding sites. In $(D)$, mutp53 is shown to associate with specific DNA elements, such as matrix attachment regions (MAR) (Gohler et al. 2005); it is proposed that this may block the recruitment of TFY to an adjacent binding site, resulting in transcriptional inhibition. Additional mechanisms most likely also exist. 
lines of biological evidence indicate that inactivation of p63/p73 can be pivotal to mutp53 GOF, since ablation of $\mathrm{p} 63 / \mathrm{p} 73$ often recapitulates the biological effects of mutp53 in an epistatic manner, whereas overexpression of some $\mathrm{p} 63 / \mathrm{p} 73$ isoforms can negate the effects of mutp53 (Adorno et al. 2009; Flores et al. 2005; Irwin et al. 2003; Lang et al. 2004; Moll et al. 2001; Olive et al. 2004). Remarkably, disruption of mutp53-p73 interactions by small peptides renders endogenous mutp53-expressing cancer cells more sensitive to killing by genotoxic anticancer drugs, whereas such peptides have no effect on cells expressing wtp53 or no p53 (Di Agostino et al. 2008).

The interaction between mutp53 and p63/ p73 can be regulated by cell-intrinsic and extrinsic signals and by additional partner proteins, as illustrated by the formation of a ternary complex between mutp53, p63, and Smad proteins, which is induced by TGF- $\beta$ and oncogenic Ras (Adorno et al. 2009). Conceivably, many other factors may modulate the association between mutp53 and p63/p73, determining the efficacy of this mechanism of mutp53 GOF.

Of note, inactivation of $\mathrm{p} 63 / \mathrm{p} 73$ by mutp 53 does not depend on the integrity of the p53 TAD (Adorno et al. 2009). This may explain why abrogation of the mitotic spindle checkpoint by mutp53, perhaps through inactivation of p73 (see previous discussion), does not require a functional TAD and can be effectively achieved by the triple mutant p53D281G,22,23 carrying two inactivating mutations in the TAD (Gualberto et al. 1998). Similarly, the TAD is dispensable for the induction of invasion and metastasis by mutp53 (Adorno et al. 2009).

In contrast, the TAD is essential for the other, p63/p73-independent mechanism of transcriptional regulation by mutp53 GOF. In this mode of action, mutp53 can physically associate with a number of sequence-specific transcription factors. Often, this enables mutp53 to "piggyback" on such factors and be brought to chromatin regions that contain DNA binding sites for those factors. In the simplest scenario, mutp53 "donates" its potent, intact TAD to the partner factor, recruiting transcriptional coactivators that are normally involved in transcriptional activation by wtp 53 , and augmenting the expression of genes that are targeted directly by the partner factor (Fig. 1). Examples of this sort include the interaction of mutp53 with NF-Y (Di Agostino et al. 2006), NF-kB (Weisz et al. 2007a), and the vitamin D3 receptor (VDR) (Stambolsky et al., unpubl. data). Alternatively, in some cases, the binding of mutp53 to a particular transcription factor may actually interfere with its functions and lead to reversal of its transcriptional effects, as exemplified by another subset of VDR-regulated genes (Stambolsky et al., unpubl. data). In yet another scenario, the binding of mutp53 to chromatin might displace a positively acting TF or recruit transcriptional corepressors to this site, in both cases leading to transrepression of adjacent genes.

Fragmentary evidence suggests that this p63/p73-independent molecular mechanism is widespread in mutp53 GOF activity. This includes studies in which biological and biochemical effects of mutp53 were found to depend on the integrity of the TAD, as well as studies showing recruitment of mutp53 to the promoters of genes whose expression it modulates, as measured by chromatin immunoprecipitation (ChIP) analysis. For instance, the antiapoptotic action of mutp53 in cancer cells is compromised by mutation of the TAD (Matas et al. 2001; Scian et al. 2005), as is also the repression of the TGF- $\beta 2$ receptor (Kalo et al. 2007) and the augmentation of VDR activity (Stambolsky et al., unpubl. data) by mutp53. Furthermore, mutp53 can be found on the promoters of the Egr1, NFKB2, TGFBR2, and Gro1 genes that it transactivates (Kalo et al. 2007; Weisz et al. 2007a; Weisz et al. 2004; Yan and Chen 2009) and of the Id2, CD95, and MSP genes that it represses (Yan et al. 2008; Zalcenstein et al. 2003; Zalcenstein et al. 2006). Recent application of genome-wide ChIP approaches to map interactions between mutp53 and chromatin is likely to reveal many additional genes whose expression is modulated by mutp53 in a similar manner (Donzelli et al. 2008).

Although direct transcriptional regulation by mutp53 and inactivation of p63/p73 are 
mechanistically distinct, it is conceivable that both mechanisms work together to exert many of the GOF effects of mutp53. One such example is the impact of mutp53 on cell migration and invasion. As discussed previously, this impact is partly caused by repression of p63regulated genes. In addition, however, mutp53 binds and transactivates the Grol gene encoding CXCL1, a chemokine that plays important roles in promoting tumor cell migration, invasion and angiogenesis by both autocrine and paracrine mechanisms (Yan and Chen 2009). Furthermore, mutp53 can promote migration by elevating the steady-state levels of the Slug protein, presumably by inhibiting p73-dependent Mdm2 gene expression, thereby blocking Mdm2-mediated Slug degradation (Wang et al. 2009). Quite certainly, future studies will identify additional modulators of these metastasis-related activities whose expression is regulated by mutp53.

Additional protein-protein interactions most certainly also play a role in mutp53 GOF. Recently, such mechanism has been implicated in the promotion of genomic instability by mutp53. Thus, it was found that mup53 can disrupt ATM-mediated cellular responses to double-stranded DNA breaks (Song et al. 2007; Song and Xu 2007). This is caused by a physical interaction between mutp53 and the nuclease Mre11, a component of the Mre11Rad50-NBS1 (MRN) complex, preventing its binding to double-stranded DNA breaks. As binding of the MRN complex to such DNA breaks is required for optimal ATM activation, the presence of mutp53 blunts this activation and enables persistence of unrepaired DNA damage. Another case in point is the interaction between p53 and topoisomerase I (Topo I). Both wtp53 and various cancer-associated mutp53 isoforms can bind Topo I and enhance its activity (Albor et al. 1998). However, given the elevated concentrations of mutp53 in cancer cells and the defective DNA damage checkpoints in cells lacking wtp53, as well as the deregulated nature of the interaction between mutp53 and Topo I (Restle et al. 2008), it is conceivable that in the case of mutp53, this interaction might lead to an increase in aberrant homologous DNA recombination events and mutagenic DNA rearrangements, spawning an additional type of genomic instability.

Another interesting protein-protein interaction of mutp53 was recently identified (Haupt et al. 2009). The promyelocytic leukemia (PML) protein is long known to possess tumor suppressor functions. Yet, when it binds to mutp53, it can augment the latter's transcriptional activity, thereby enhancing the ability of mutp53 to stimulate cell proliferation and colony formation. One implication of this finding is that although PML is a tumor suppressor in cells with functional wtp53, in which it interacts with wtp53 and contributes to its activation in response to genotoxic stress, it can be converted into a cancer-promoting protein on binding to mutp53. It is very likely that many additional proteins that normally bind p53 and facilitate its activation will operate similarly on mutp53, except that the biological outcome and impact on tumor progression will be totally opposite.

\section{STABILIZATION OF MUTANT p53 IN CANCER CELLS - A KEY TO GOF?}

Efficient GOF action by mutp53 requires elevated levels of mutp53 protein in the affected cell. Although transcriptional and translational mechanisms are likely to contribute to such elevation, its main driving mechanism is believed to be the increased protein stability of mutp53. Thus, whereas wtp53 is generally observed to be short lived, owing to its efficient degradation by the ubiquitin-proteasome pathway, mutp53 is often found to be rather stable. Initially, this was suspected to be a salient feature of mutp53, directly caused by the impact of the mutations on the biochemical properties of the mutant protein. However, subsequent work revealed that mutp53 is not intrinsically stable; rather, changes that occur within tumor cells result in its stabilization. Thus, in primary cells derived from human Li-Fraumeni syndrome patients, who carry germline p53 mutations, the levels of mutp53 are rather low and comparable to those of wtp53 (Yin et al. 1992). Similarly, mutp53 protein levels are low 
in mutp53 knockin mice, but increase substantially in a fraction of tumors that emerge in such mice (Lang et al. 2004; Terzian et al. 2008). Indeed, early experiments already indicated that a mouse mutp53 is degraded faster in nontransformed fibroblasts than in transformed ones (Halevy et al. 1989).

So how is mutp53 converted from an unstable to a very stable protein? The degradation of wtp53 is achieved through a multitude of mechanisms, involving several E3 ubiquitin ligases that target p53 for polyubiquitylation and consequent proteasomal degradation, as well as ubiquitin-independent degradation in the proteasome. Of those, the best studied and probably the most important driver of p53 degradation is $\mathrm{Mdm} 2$, an E3 ligase that promotes p53 ubiquitylation as well as postubiquitylation steps in p53 degradation, and also represses p53 mRNA translation. Escape from Mdm2-mediated degradation is therefore an appealing mechanism for mutp53 stabilization. However, mutp53 is still susceptible to Mdm2mediated degradation (Haupt et al. 1997; Lukashchuk and Vousden 2007), arguing that the mutations per se do not render p53 immune to the action of Mdm2. Instead, other mechanisms must be at work to enable p53 stabilization in tumor cells.

The simplest explanation for mutp53 stabilization draws on the fact that cancer-associated mutp53 isoforms lack the ability to transactivate wtp53 target genes. The $M d m 2$ gene is a classical positive transcriptional target of wtp53, and this drives a negative feedback loop that helps maintain wtp53 levels very low in unstressed cells. However, mutp53 fails to transactivate the $M d m 2$ gene. Hence, Mdm2 protein levels are likely to be rather low in cells that express only mutp53. Indeed, ablation of endogenous Mdm2 in mutp53 knockin mice leads to a substantial increase in endogenous mutp53 levels (Terzian et al. 2008). Importantly, this results in an earlier age of tumor onset and a GOF metastatic phenotype.

Yet, reduced levels of Mdm2 in mutp53expressing cells are most probably not the sole mechanism that can enable mutp53 stabilization. This is revealed even in the previous knockin model, in which a similar degree of p53 protein accumulation could be achieved also by ablation of the p16INK4a tumor suppressor locus (Terzian et al. 2008). A clue to the nature of the additional mutp53-stabilizing mechanism may be provided by the fact that, in primary cells derived from mutp53 knockin mice, the intrinsically unstable mutp53 can be stabilized by genotoxic stress, very much like wtp53 (Lang et al. 2004). Because many tumor cells experience chronic DNA damage, it is conceivable that the resultant signaling events, normally aimed at stabilizing and activating the endogenous wtp53, do the same also to the resident mutp53, except that rather than curbing cancer, this now results in cancer promotion. As one interesting example of the difference between cells containing wtp53 and mutp53, $\mathrm{Li}$ and coworkers reported that PTEN, a potent tumor suppressor whose inactivation in cancer is almost as frequent as that of p53, might actually exert tumor-promoting properties when assayed on the background of p53 GOF mutations (Li et al. 2008). This was found to be caused by the ability of PTEN to enhance mutp53 protein levels via inhibition of its degradation by $\mathrm{Mdm} 2$ and possibly also via direct protein binding. Further work is likely to reveal additional mechanisms that lead to mutp53 stabilization in cancer cells, thereby fueling its GOF effects.

\section{CONCLUDING REMARKS}

As is apparent from this article, the accrual of p53 mutations equips emerging tumor cells with much more than just the loss of wtp53 tumor suppressor function. This poses mutp53 as a valid target for inactivation by prospective anticancer therapies. Ideally, molecules that cause mutp53 to regain wtp53 activity bear the most promise, as they are expected to exert a dual effect: reinstate wtp53 tumor suppressor function, and rid the cell of mutp53 GOF. A number of such molecules have already been identified and described, and shown to exert antitumor effects in experimental mouse models (Bykov et al. 2002; Bykov et al. 2005; Foster et al. 1999; Lambert et al. 2009). Yet, approaches 
M. Oren and V. Rotter

that are more directly aimed toward elimination of mutp53, such as therapeutic administration of p53 siRNA to cancer patients whose tumors exhibit high mutp53 expression, are also worthy of consideration. Moreover, one may attempt to target proteins whose expression is markedly up-regulated by mutp53, particularly in cases in which such proteins are "druggable" targets, such as transmembrane or secreted proteins or enzymes whose catalytic activity is susceptible to inhibition by small molecular weight compounds. The future will tell to which extent the knowledge on mutp53 GOF, as well as the tools developed through this knowledge, can eventually benefit cancer patients.

\section{ACKNOWLEDGMENTS}

Work in the authors' laboratories is supported by grant R37 CA40099 from the National Cancer Institute, a Center of Excellence grant from the Flight Attendant Medical Research Institute, grants from the European Commission (Mutp53, FP6 Contract 502983 and OncomiRs, FP7 Contract 201102), and the Robert Bosch Foundation. M.O. and V.R. are the incumbents of the Andre Lwoff Professorial Chair in Molecular Biology and the Norman and Helen Asher Professorial Chair for Cancer Research, respectively, at the Weizmann institute.

\section{REFERENCES}

Adorno M, Cordenonsi M, Montagner M, Dupont S, Wong C, Hann B, Solari A, Bobisse S, Rondina MB, Guzzardo V, et al. 2009. A Mutant-p53/Smad complex opposes p63 to empower TGF $\beta$-induced metastasis. Cell 137: 87-98.

Albor A, Kaku S, Kulesz-Martin M. 1998. Wild-type and mutant forms of p53 activate human topoisomerase I: a possible mechanism for gain of function in mutants. Cancer Res 58: 2091-2094.

Beckerman R, Prives C. 2010. Transcriptional regulation by p53. Cold Spring Harb Perspect Biol 2: a000935.

Blandino G, Levine AJ, Oren M. 1999. Mutant p53 gain of function: differential effects of different p53 mutants on resistance of cultured cells to chemotherapy. Oncogene 18: $477-485$.

Bossi G, Lapi E, Strano S, Rinaldo C, Blandino G, Sacchi A. 2006. Mutant p53 gain of function: reduction of tumor malignancy of human cancer cell lines through abrogation of mutant p53 expression. Oncogene 25: 304-309.

Bossi G, Marampon F, Maor-Aloni R, Zani B, Rotter V, Oren M, Strano S, Blandino G, Sacchi A. 2008. Conditional
RNA interference in vivo to study mutant p53 oncogenic gain of function on tumor malignancy. Cell Cycle (Georgetown, Tex) 7: 1870-1879.

Brosh R, Rotter V. 2009. When mutants gain new powers: news from the mutant p53 field. Nature Rev Cancer (in press).

Buganim Y, Kalo E, Brosh R, Besserglick H, Nachmany I, Rais Y, Stambolsky P, Tang X, Milyavsky M, Shats I, et al. 2006. Mutant p53 protects cells from 12-Otetradecanoylphorbol-13-acetate-induced death by attenuating activating transcription factor 3 induction. Cancer Res 66: 10750-10759.

Bykov VJ, Issaeva N, Shilov A, Hultcrantz M, Pugacheva E, Chumakov P, Bergman J, Wiman KG, Selivanova G. 2002. Restoration of the tumor suppressor function to mutant p53 by a low-molecular-weight compound. Nature Medicine 8: 282-288.

Bykov VJ, Issaeva N, Zache N, Shilov A, Hultcrantz M, Bergman J, Selivanova G, Wiman KG. 2005. Reactivation of mutant p53 and induction of apoptosis in human tumor cells by maleimide analogs. J Biol Chem 280: 30384-30391.

Caulin C, Nguyen T, Lang GA, Goepfert TM, Brinkley BR, Cai WW, Lozano G, Roop DR. 2007. An inducible mouse model for skin cancer reveals distinct roles for gainand loss-of-function p53 mutations. J Clin Invest 117: 1893-1901.

Chin KV, Ueda K, Pastan I, Gottesman MM. 1992. Modulation of activity of the promoter of the human MDR1 gene by Ras and p53. Science 255: 459-462.

Cordenonsi M, Dupont S, Maretto S, Insinga A, Imbriano C, Piccolo S. 2003. Links between tumor suppressors: p53 is required for TGF- $\beta$ gene responses by cooperating with Smads. Cell 113: 301-314.

Deb S, Jackson CT, Subler MA, Martin DW. 1992. Modulation of cellular and viral promoters by mutant human p53 proteins found in tumor cells. J Virol 66: 6164-6170.

Deb D, Scian M, Roth KE, Li W, Keiger J, Chakraborti AS, Deb SP, Deb S. 2002. Hetero-oligomerization does not compromise 'gain of function' of tumor-derived p53 mutants. Oncogene 21: 176-189.

Derynck R, Akhurst RJ. 2007. Differentiation plasticity regulated by TGF- $\beta$ family proteins in development and disease. Nat Cell Biol 9: 1000-1004.

Derynck R, Akhurst RJ, Balmain A. 2001. TGF- $\beta$ signaling in tumor suppression and cancer progression. Nature Genetics 29: 117-129.

Di Agostino S, Cortese G, Monti O, Dell'Orso S, Sacchi A, Eisenstein M, Citro G, Strano S, Blandino G. 2008. The disruption of the protein complex mutantp53/p73 increases selectively the response of tumor cells to anticancer drugs. Cell Cycle (Georgetown, Tex) 7: 3440-3447.

Di Agostino S, Strano S, Emiliozzi V, Zerbini V, Mottolese M, Sacchi A, Blandino G, Piaggio G. 2006. Gain of function of mutant p53: The mutant p53/NF-Y protein complex reveals an aberrant transcriptional mechanism of cell cycle regulation. Cancer Cell 10: 191-202.

Dittmer D, Pati S, Zambetti G, Chu S, Teresky AK, Moore M, Finlay C, Levine AJ. 1993. Gain of function mutations in p53. Nature Genetics 4: 42-46. 
Dong P, Tada M, Hamada J, Nakamura A, Moriuchi T, Sakuragi N. 2007. p53 dominant-negative mutant $\mathrm{R} 273 \mathrm{H}$ promotes invasion and migration of human endometrial cancer HHUA cells. Clin Exp Metastasis 24: 471-483.

Donzelli S, Biagioni F, Fausti F, Strano S, Fontemaggi G, Blandino G. 2008. Oncogenomic Approaches in Exploring Gain of Function of Mutant p53. Current Genomics 9: 200-207.

Duan W, Ding H, Subler MA, Zhu WG, Zhang H, Stoner GD, Windle JJ, Otterson GA, Villalona-Calero MA. 2002. Lung-specific expression of human mutant p53-273H is associated with a high frequency of lung adenocarcinoma in transgenic mice. Oncogene 21: $7831-7838$.

Duan W, Gao L, Jin D, Otterson GA, Villalona-Calero MA. 2008. Lung specific expression of a human mutant p53 affects cell proliferation in transgenic mice. Transgenic Research 17: 355-366.

Duan W, Gao L, Wu X, Hade EM, Gao JX, Ding H, Barsky SH, Otterson GA, Villalona-Calero MA. 2009. Expression of a mutant p53 results in an age-related demographic shift in spontaneous lung tumor formation in transgenic mice. PloS One 4: e5563.

El-Hizawi S, Lagowski JP, Kulesz-Martin M, Albor A. 2002. Induction of gene amplification as a gain-of-function phenotype of mutant p53 proteins. Cancer Res 62: $3264-3270$.

Flores ER, Sengupta S, Miller JB, Newman JJ, Bronson R, Crowley D, Yang A, McKeon F, Jacks T. 2005. Tumor predisposition in mice mutant for $\mathrm{p} 63$ and p 73 : Evidence for broader tumor suppressor functions for the p53 family. Cancer Cell 7: 363-373.

Foster BA, Coffey HA, Morin MJ, Rastinejad F. 1999. Pharmacological rescue of mutant p53 conformation and function. Science 286: 2507-2510

Frazier MW, He X, Wang J, Gu Z, Cleveland JL, Zambetti GP. 1998. Activation of c-myc gene expression by tumorderived p53 mutants requires a discrete C-terminal domain. Mol Cell Biol 18: 3735-3743.

Gaiddon C, Lokshin M, Ahn J, Zhang T, Prives C. 2001. A subset of tumor-derived mutant forms of p53 downregulate p63 and p73 through a direct interaction with the p53 core domain. Mol Cell Biol 21: 1874-1887.

Gohler T, Jager S, Warnecke G, Yasuda H, Kim E, Deppert W. 2005. Mutant p53 proteins bind DNA in a DNA structure-selective mode. Nucleic Acids Res 33: 1087-1100.

Gualberto A, Aldape K, Kozakiewicz K, Tlsty TD. 1998. An oncogenic form of p53 confers a dominant, gainof-function phenotype that disrupts spindle checkpoint control. Proc Natl Acad Sci U S A 95: 5166-5171.

Gurova KV, Rokhlin OW, Budanov AV, Burdelya LG, Chumakov PM, Cohen MB, Gudkov AV. 2003. Cooperation of two mutant p53 alleles contributes to Fas resistance of prostate carcinoma cells. Cancer Res 63: 2905-2912.

Halevy O, Hall A, Oren M. 1989. Stabilization of the p53 transformation-related protein in mouse fibrosarcoma cell lines: Effects of protein sequence and intracellular environment. Mol Cell Biol 9: 3385-3392.
Halevy O, Rodel J, Peled A, Oren M. 1991. Frequent p53 mutations in chemically induced murine fibrosarcoma. Oncogene 6: 1593-1600.

Haupt S, di Agostino S, Mizrahi I, Alsheich-Bartok O, Voorhoeve M, Damalas A, Blandino G, Haupt Y. 2009. Promyelocytic leukemia protein is required for gain of function by mutant 53. Cancer Res 69: 4818-4826.

Haupt Y, Maya R, Kazaz A, Oren M. 1997. Mdm2 promotes the rapid degradation of 53. Nature 387: 296-299.

Heinlein C, Krepulat F, Lohler J, Speidel D, Deppert W, Tolstonog GV. 2008. Mutant p53(R270H) gain of function phenotype in a mouse model for oncogeneinduced mammary carcinogenesis. Int J Cancer 122: 1701-1709.

Hingorani SR, Wang L, Multani AS, Combs C, Deramaudt TB, Hruban RH, Rustgi AK, Chang S, Tuveson DA. 2005. Trp53R172H and KrasG12D cooperate to promote chromosomal instability and widely metastatic pancreatic ductal adenocarcinoma in mice. Cancer Cell 7: 469-483.

Hsiao M, Low J, Dorn E, Ku D, Pattengale P, Yeargin J, Haas M. 1994. Gain-of-function mutations of the p53 gene induce lymphohematopoietic metastatic potential and tissue invasiveness. Am J Pathol 145: 702-714.

Irwin MS, Kondo K, Marin MC, Cheng LS, Hahn WC, Kaelin WG Jr. 2003. Chemosensitivity linked to p73 function. Cancer Cell 3: 403-410.

Iwamoto KS, Mizuno T, Ito T, Tsuyama N, Kyoizumi S, Seyama T. 1996. Gain-of-function p53 mutations enhance alteration of the T-cell receptor following $\mathrm{X}$-irradiation, independently of the cell cycle and cell survival. Cancer Res 56: 3862-3865.

Joerger AC, Fersht AR. 2007. Structural biology of the tumor suppressor p53 and cancer-associated mutants. Advances in cancer research 97: 1-23.

Joerger AC, Fersht AR. 2008. Structural biology of the tumor suppressor p53. Annual review of biochemistry 77: $557-582$.

Kalo E, Buganim Y, Shapira KE, Besserglick H, Goldfinger N, Weisz L, Stambolsky P, Henis YI, Rotter V. 2007. Mutant p53 attenuates the SMAD-dependent transforming growth factor $\beta 1$ (TGF- $\beta 1$ ) signaling pathway by repressing the expression of TGF- $\beta$ receptor type II. Mol Cell Biol 27: 8228-8242.

Knaup KX, Roemer K. 2004. Cell type-specific regulation of calmodulin 2 expression by mutant p53. FEBS Lett 569: $70-74$.

Lambert JM, Gorzov P, Veprintsev DB, Soderqvist M, Segerback D, Bergman J, Fersht AR, Hainaut P, Wiman KG, Bykov V.J. 2009. PRIMA-1 reactivates mutant p53 by covalent binding to the core domain. Cancer Cell 15: 376-388.

Lang GA, Iwakuma T, Suh YA, Liu G, Rao VA, Parant JM, Valentin-Vega YA, Terzian T, Caldwell LC, Strong LC, et al. 2004. Gain of function of a p53 hot spot mutation in a mouse model of Li-Fraumeni syndrome. Cell 119: $861-872$.

Lanyi A, Deb D, Seymour RC, Ludes-Meyers JH, Subler MA, Deb S. 1998. 'Gain of function' phenotype of tumor-derived mutant p53 requires the oligomerization/nonsequence-specific nucleic acid-binding domain. Oncogene 16: 3169-3176. 


\section{Oren and V. Rotter}

Lavra L, Ulivieri A, Rinaldo C, Dominici R, Volante M, Luciani E, Bartolazzi A, Frasca F, Soddu S, Sciacchitano S. 2009. Gal-3 is stimulated by gain-of-function p53 mutations and modulates chemoresistance in anaplastic thyroid carcinomas. J Pathol 218: 66-75.

Lee YI, Lee S, Das GC, Park US, Park SM, Lee YI. 2000. Activation of the insulin-like growth factor II transcription by aflatoxin B1 induced p53 mutant 249 is caused by activation of transcription complexes; implications for a gain-of-function during the formation of hepatocellular carcinoma. Oncogene 19: 3717-3726.

Levine AJ, Oren M. 2009. The first 30 years of p53: growing ever more complex. Nat Rev Cancer 9: 749-758.

Li Y, Guessous F, Kwon S, Kumar M, Ibidapo O, Fuller L, Johnson E, Lal B, Hussaini I, Bao Y, et al. 2008 PTEN has tumor-promoting properties in the setting of gain-of-function p53 mutations. Cancer Res 68: $1723-1731$.

Li R, Sutphin PD, Schwartz D, Matas D, Almog N, Wolkowicz R, Goldfinger N, Pei H, Prokocimer M, Rotter V. 1998. Mutant p53 protein expression interferes with p53-independent apoptotic pathways. Oncogene 16: 3269-3277.

Loging WT, Reisman D. 1999. Elevated expression of ribosomal protein genes L37, RPP-1, S2 in the presence of mutant p53. Cancer Epidemiol Biomarkers Prev 8: 1011-1016.

Lotem J, Sachs L. 1995. A mutant p53 antagonizes the deregulated c-myc-mediated enhancement of apoptosis and decrease in leukemogenicity. Proc Natl Acad Sci U S A 92: 9672-9676.

Lozano G. 2007. The oncogenic roles of p53 mutants in mouse models. Current opinion in genetics \& development 17: $66-70$

Ludes-Meyers JH, Subler MA, Shivakumar CV, Munoz RM, Jiang P, Bigger JE, Brown DR, Deb SP, Deb S. 1996. Transcriptional activation of the human epidermal growth factor receptor promoter by human 53. Mol Cell Bio 16: 6009-6019.

Lukashchuk N, Vousden KH. 2007. Ubiquitination and degradation of mutant p53. Mol Cell Biol 27: 8284-8295.

Malkin D, Li FP, Strong LC, Fraumeni JF Jr, Nelson CE, Kim DH, Kassel J, Gryka MA, Bischoff FZ, Tainsky MA, et al. 1990. Germ line p53 mutations in a familial syndrome of breast cancer, sarcomas, other neoplasms. Science 250: $1233-1238$.

Marin MC, Jost CA, Brooks LA, Irwin MS, O’Nions J, Tidy JA, James N, McGregor JM, Harwood CA, Yulug IG, et al. 2000. A common polymorphism acts as an intragenic modifier of mutant p53 behaviour. Nature Genetics 25: $47-54$.

Massague J. 2008. TGF $\beta$ in Cancer. Cell 134: 215-230.

Matas D, Sigal A, Stambolsky P, Milyavsky M, Weisz L, Schwartz D, Goldfinger N, Rotter V. 2001. Integrity of the $\mathrm{N}$-terminal transcription domain of p53 is required for mutant p53 interference with drug-induced apoptosis. Embo J 20: 4163 - 4172.

Michalovitz D, Halevy O, Oren M. 1991. p53 mutations: gains or losses? J Cell Bioch 45: 22-29.
Moll UM, Erster S, Zaika A. 2001. p53, p63 and p73-solos, alliances and feuds among family members. Biochim Biophys Acta 1552: 47-59.

Murphy M, Ahn J, Walker KK, Hoffman WH, Evans RM, Levine AJ, George DL. 1999. Transcriptional repression by wild-type p53 utilizes histone deacetylases, mediated by interaction with mSin3a. Genes Dev 13: 2490-2501.

Murphy KL, Dennis AP, Rosen JM. 2000. A gain of function p53 mutant promotes both genomic instability and cell survival in a novel p53-null mammary epithelial cell model. Faseb J 14: 2291-2302.

Offer H, Wolkowicz R, Matas D, Blumenstein S, Livneh Z, Rotter V. 1999. Direct involvement of p53 in the base excision repair pathway of the DNA repair machinery. FEBS Lett 450: 197-204.

Olive KP, Tuveson DA, Ruhe ZC, Yin B, Willis NA, Bronson RT, Crowley D, Jacks T. 2004. Mutant p53 gain of function in two mouse models of Li-Fraumeni syndrome. Cell 119: $847-860$.

Olivier M, Petitjean A, Marcel V, Petre A, Mounawar M, Plymoth A, de Fromentel CC, Hainaut P. 2009. Recent advances in p53 research: An interdisciplinary perspective. Cancer Gene Therapy 16: 1-12.

Olivier M, Hollstein M, Hainaut P. 2010. TP53 mutations in human cancers: origins, consequences, and clinical use. Cold Spring Harb Perspect Biol 2: a001008.

Peart MJ, Prives C. 2006. Mutant p53 gain of function: The NF-Y connection. Cancer Cell 10: 173-174.

Peled A, Zipori D, Rotter V. 1996. Cooperation between p53-dependent and p53-independent apoptotic pathways in myeloid cells. Cancer Res 56: 2148-2156.

Petitjean A, Achatz MI, Borresen-Dale AL, Hainaut P, Olivier M. 2007. TP53 mutations in human cancers: Functional selection and impact on cancer prognosis and outcomes. Oncogene 26: 2157-2165.

Pohl J, Goldfinger N, Radler-Pohl A, Rotter V, Schirrmacher V. 1988. p53 increases experimental metastatic capacity of murine carcinoma cells. Mol Cell Biol 8: 2078-2081.

Restle A, Farber M, Baumann C, Bohringer M, Scheidtmann KH, Muller-Tidow C, Wiesmuller L. 2008. Dissecting the role of p53 phosphorylation in homologous recombination provides new clues for gain-of-function mutants. Nucleic Acids Res 36: 5362-5375.

Rotter V. 1983. p53, a transformation-related cellularencoded protein, can be used as a biochemical marker for the detection of primary mouse tumor cells. Proc Natl Acad Sci 80: 2613-2617.

Scian MJ, Stagliano KE, Anderson MA, Hassan S, Bowman M, Miles MF, Deb SP, Deb S. 2005. Tumor-derived p53 mutants induce NF-kappaB2 gene expression. Mol Cell Biol 25: 10097-10110.

Scian MJ, Stagliano KE, Deb D, Ellis MA, Carchman EH, Das A, Valerie K, Deb SP, Deb S. 2004a. Tumor-derived p53 mutants induce oncogenesis by transactivating growth-promoting genes. Oncogene 23: 4430-4443.

Scian MJ, Stagliano KE, Ellis MA, Hassan S, Bowman M, Miles MF, Deb SP, Deb S. 2004b. Modulation of gene expression by tumor-derived p53 mutants. Cancer Res 64: 7447-7454. 
Sigal A, Rotter V. 2000. Oncogenic mutations of the p53 tumor suppressor: The demons of the guardian of the genome. Cancer Res 60: 6788-6793.

Singer S, Ehemann V, Brauckhoff A, Keith M, Vreden S, Schirmacher P, Breuhahn K. 2007. Protumorigenic overexpression of stathmin/Op18 by gain-of-function mutation in p53 in human hepatocarcinogenesis. Hepatology (Baltimore, Md) 46: 759-768.

Song H, Xu Y. 2007. Gain of function of p53 cancer mutants in disrupting critical DNA damage response pathways. Cell Cycle (Georgetown, Tex) 6: 1570-1573.

Song H, Hollstein M, Xu Y. 2007. p53 gain-of-function cancer mutants induce genetic instability by inactivating ATM. Nat Cell Biol 9: 573-580.

Srivastava S, Zou ZQ, Pirollo K, Blattner W, Chang EH. 1990. Germ-line transmission of a mutated p53 gene in a cancer-prone family with Li-Fraumeni syndrome. Nature 348: 747-749.

Strano S, Dell'Orso S, Di Agostino S, Fontemaggi G, Sacch A, Blandino G. 2007. Mutant p53: An oncogenic transcription factor. Oncogene 26: 2212-2219.

Strano S, Fontemaggi G, Costanzo A, Rizzo MG, Monti O, Baccarini A, Del Sal G, Levrero M, Sacchi A, Oren M, et al. 2002. Physical interaction with human tumorderived p53 mutants inhibits p63 activities. J Biol Chem 277: 18817-18826.

Talos F, Nemajerova A, Flores ER, Petrenko O, Moll UM 2007. p73 suppresses polyploidy and aneuploidy in the absence of functional p53. Molecular Cell 27: 647-659.

Taylor WR, Egan SE, Mowat M, Greenberg AH, Wright J.A. 1992. Evidence for synergistic interactions between ras, myc and a mutant form of p53 in cellular transformation and tumor dissemination. Oncogene 7: 1383-1390.

Terzian T, Suh YA, Iwakuma T, Post SM, Neumann M, Lang GA, Van Pelt CS, Lozano G. 2008. The inherent instability of mutant p53 is alleviated by Mdm2 or p16INK4a loss. Genes Dev 22: 1337-1344.

Vikhanskaya F, Lee MK, Mazzoletti M, Broggini M, Sabapathy K. 2007. Cancer-derived p53 mutants suppress p53-target gene expression-potential mechanism for gain of function of mutant p53. Nucleic Acids Res 35: 2093-2104.

Vogelstein B, Kinzler KW. 1993. The multistep nature of cancer. Trends Genet 9: 138-141.

Wang SP, Wang WL, Chang YL, Wu CT, Chao YC, Kao SH, Yuan A, Lin CW, Yang SC, Chan WK, et al. 2009. p53 controls cancer cell invasion by inducing the MDM2mediated degradation of Slug. Nat Cell Biol 11: 694-704.
Weisz L, Oren M, Rotter V. 2007b. Transcription regulation by mutant p53. Oncogene 26: 2202-2211.

Weisz L, Damalas A, Liontos M, Karakaidos P, Fontemaggi G, Maor-Aloni R, Kalis M, Levrero M, Strano S, Gorgoulis VG, et al. 2007a. Mutant p53 enhances nuclear factor kappaB activation by tumor necrosis factor $\alpha$ in cancer cells. Cancer Res 67: 2396-2401.

Weisz L, Zalcenstein A, Stambolsky P, Cohen Y, Goldfinger N, Oren M, Rotter V. 2004. Transactivation of the EGR1 gene contributes to mutant $\mathrm{p} 53$ gain of function. Cancer Res 64: 8318-8327.

Werner H, Karnieli E, Rauscher FJ, LeRoith D. 1996. Wildtype and mutant p53 differentially regulate transcription of the insulin-like growth factor I receptor gene. Proc Natl Acad Sci 93: 8318-8323.

Wolf D, Harris N, Rotter V. 1984. Reconstitution of p53 expression in a nonproducer Ab-MuLV-transformed cell line by transfection of a functional p53 gene. Cell 38: $119-126$.

Wong RP, Tsang WP, Chau PY, Co NN, Tsang TY, Kwok TT. 2007. p53-R273H gains new function in induction of drug resistance through down-regulation of procaspase-3. Molecular Cancer Therapeutics 6: 1054-1061.

Yan W, Chen X. 2009. Identification of GRO1 as a critical determinant for mutant p53 gain of function. J Biol Chem 284: $12178-12187$.

Yan W, Liu G, Scoumanne A, Chen X. 2008. Suppression of inhibitor of differentiation 2, a target of mutant p53, is required for gain-of-function mutations. Cancer Res 68: 6789-6796.

Yap DB, Hsieh JK, Zhong S, Heath V, Gusterson B, Crook T, $\mathrm{Lu}$ X. 2004. Ser392 phosphorylation regulates the oncogenic function of mutant p53. Cancer Res 64: $4749-4754$.

Yin Y, Tainsky MA, Bischoff FZ, Strong LC, Wahl GM. 1992. Wild-type p53 restores cell cycle control and inhibits gene amplification in cells with mutant p53 alleles. Cell 70: 937-948.

Zalcenstein A, Stambolsky P, Weisz L, Muller M, Wallach D, Goncharov TM, Krammer PH, Rotter V, Oren M. 2003. Mutant p53 gain of function: Repression of CD95(Fas/ APO-1) gene expression by tumor-associated p53 mutants. Oncogene 22: 5667-5676.

Zalcenstein A, Weisz L, Stambolsky P, Bar J, Rotter V, Oren M. 2006. Repression of the MSP/MST-1 gene contributes to the antiapoptotic gain of function of mutant p53. Oncogene 25: 359-369. 


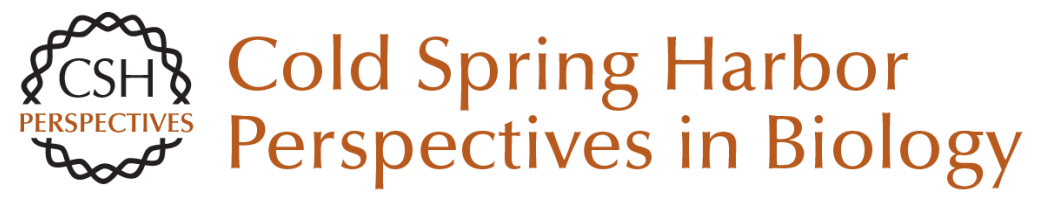

\section{Mutant p53 Gain-of-Function in Cancer}

Moshe Oren and Varda Rotter

Cold Spring Harb Perspect Biol 2010; doi: 10.1101/cshperspect.a001107 originally published online December 16, 2009

\section{Subject Collection The p53 Family}

The Origins and Evolution of the p53 Family of Genes

Vladimir A. Belyi, Prashanth Ak, Elke Markert, et al.

Mouse Models of p53 Functions Guillermina Lozano

TP53 Mutations in Human Cancers: Origins, Consequences, and Clinical Use

Magali Olivier, Monica Hollstein and Pierre Hainaut

p53 Research: The Past Thirty Years and the Next Thirty Years

David Lane and Arnold Levine

Transcriptional Regulation by P53

Rachel Beckerman and Carol Prives

p53-based Cancer Therapy

David P. Lane, Chit Fang Cheok and Sonia Lain

Phylogeny and Function of the Invertebrate p53

Superfamily

Rachael Rutkowski, Kay Hofmann and Anton Gartner

Tied Up in Loops: Positive and Negative

Autoregulation of p53

Xin Lu
The Tumor Suppressor p53: From Structures to

Drug Discovery

Andreas C. Joerger and Alan R. Fersht

p53 Regulation of Metabolic Pathways

Eyal Gottlieb and Karen H. Vousden

The Regulation of the p53-mediated Stress

Response by MDM2 and MDM4 Mary Ellen Perry

Zebrafish Models of p53 Functions Narie Y. Storer and Leonard I. Zon

p63 and p73, the Ancestors of p53

V. Dötsch, F. Bernassola, D. Coutandin, et al.

Pathologies Associated with the p53 Response Andrei V. Gudkov and Elena A. Komarova

Single-nucleotide Polymorphisms in the p53

Signaling Pathway

Lukasz F. Grochola, Jorge Zeron-Medina, Sophie Mériaux, et al.

Clinical Outcomes and Correlates of TP53

Mutations and Cancer

Ana I. Robles and Curtis C. Harris

For additional articles in this collection, see http://cshperspectives.cshlp.org/cgi/collection/

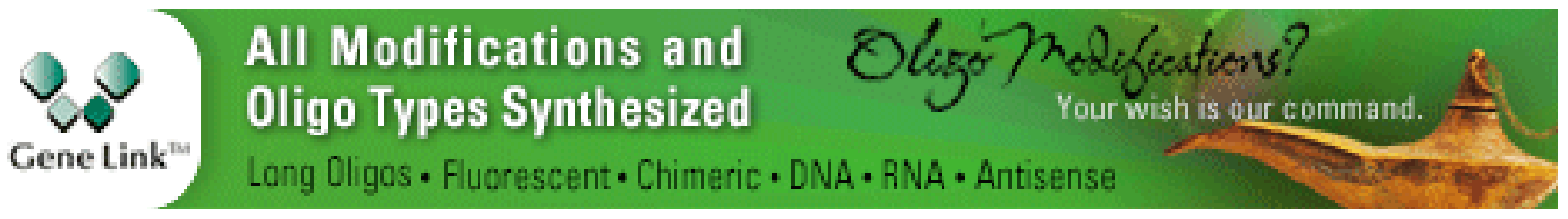

Copyright @ 2010 Cold Spring Harbor Laboratory Press; all rights reserved 